\title{
Vier unveröffentlichte Briefe Dorothea Veits und Friedrich Schlegels aus dem Jahr I 802
}

In dem Maße, in dem die Arbeit an der Kritischen Friedrich-SchlegelAusgabe (KFSA) fortschreitet, werden auch bislang unveröffentlichte Dokumente entdeckt. Das gilt insbesondere für den Briefwechsel Friedrich Schlegels und Dorothea Schlegel-Veits, dessen Edition den größten Anteil der ausstehenden Arbeiten ausmacht. Im Folgenden sollen vier neu entdeckte Texte aus der Pariser Zeit Friedrich und Dorothea Schlegel-Veits erstmals veröffentlich werden. Sie stammen allesamt aus dem Jahr I 802.

Zwei davon betreffen den schriftlichen Austausch zwischen damals noch Dorothea Veit bzw. Friedrich Schlegel einerseits und Helmina von Hastfer, die seit Paris eine ihrer wichtigsten persönlichen Briefpartnerinnen (insbesondere Dorotheas) blieb, andererseits. Beide Briefe waren zwar bekannt, bisher aber nur in Form von Abschriften, die Franziska von Longard angefertigt hatte. Die Abschriften befinden sich in Sigmaringen und sind Bestandteil des Privatarchivs von HansJoachim Dopfer, der sie dankenswerterweise zur Verfügung gestellt hat. Inzwischen konnten auch die Originale aufgefunden werden, die in der Biblioteka Jagiellońska der Uniwersytet Jagielloński in Kraków liegen. Sie gehören dort zur Sammlung Varnhagen. Mit der freundlichen Einwilligung der Biblioteka Jagiellońska wird daher die Handschrift der ersten beiden Briefe der folgenden Edition zugrunde gelegt.

Die Briefe drei und vier entstammen der Korrespondenz Friedrich Schlegels mit seinem damals wichtigsten Verleger Friedrich Wilmans, für dessen Verlag Schlegel die Europa herausgab. Der erste Brief vom 22. Oktober 1802 befindet sich im Goethe-Schiller-Archiv (Weimar). Aus dem Nachlass Ernst Behlers stammt eine Fotokopie der Handschrift des zweiten Briefes von Friedrich Schlegel an Friedrich Wilmans vom 3 I. Dezember I 802; leider konnte für diesen Brief der Aufbewahrungsort der Originalhandschrift noch nicht ermittelt werden.

Nach kurzer inhaltlicher Erläuterung folgt jeweils der Abdruck des Briefes mit einem kritischen Apparat sowie einem kurzen Über- 
lieferungsbericht. Auf einen Zeilenkommentar, wie ihn die KFSA bieten wird, musste aus Platzgründen verzichtet werden. Der kritische Apparat ${ }^{1}$ sowie der Überlieferungsbericht sind den Editionsrichtlinien der KFSA angenähert, aber nicht in allen Einzelheiten mit ihnen identisch. ${ }^{2}$

I. Dorothea Veit an Helmina von Hastfer; vermutlich I 9. Juli I 802

\section{I.I Erläuterung}

Helmina von Hastfer, geb. von Klencke (1753-1 856), Enkelin der Karschin, ab i 805 Helmina von Chézy, war nach ihrer gescheiterten ersten Ehe I 80 I auf Betreiben der französischen Emigrantin und Schriftstellerin Mme de Genlis (I 746- I 830) von Berlin nach Paris gekommen und hatte zunächst bei dieser gewohnt. ${ }^{3}$ Im Juli I 802 verschlechterten sich nach ihren eigenen Angaben die persönlichen Beziehungen zu Mme de Genlis aber so sehr, dass Helmina von Hastfer nach einer neuen Unterkunft Ausschau hielt, die sie bis etwa Ende September 1802 bei dem alternden Schweizer Schöngeist Baron d'Escherny (1733-I 8 I 5), der in der Schlusszeile auch genannt wird, auf dessen Besitzung in Versailles (Z. 37, 50) fand.

$\mathrm{Zu}$ dieser Zeit war sie bereits mit dem jungen Publizisten und Altphilologen Johann Gottlieb Schweighäuser (Z. 37) verlobt. Die-

1 Folgende diakritische Zeichen werden verwendet: |= Seitenwechsel; KAPITÄLCHEN $=$ lateinische Schrift in Handschrift $(\mathrm{H}) .-[\ldots]=$ Eingriffe des Herausgebers; $[\times]$, $[\times x]=$ verderbter Text, Textverlust; $a b c \sim=$ Unsichere Lesart; $\langle a b c\rangle=$ gestrichener Text; $\langle a b c\rangle\langle a b c:=$ Textersatz durch Überschreibung; $\langle x x\rangle,\langle x \times x\rangle$ $=$ Texttilgung durch den Autor; $[\mathrm{abc}\rceil=$ Einfügung des Autors über der Zeile.

2 Vgl. Matthias Emrich: »Abschluss der Kritischen Friedrich-Schlegel-Ausgabe «. In: Athenäum 22 (2012), S. I 39-1 43.

3 Auf den biographischen Hintergrund, der den Bezugspunkt für die Erläuterungen zu den beiden Briefen an Helmina von Hastfer bildet, kann hier aus Raumgründen nur summarisch hingewiesen werden: Helmina von Chézy: »Überlieferungen und Umrisse aus den Tagen Napoleons «. In: Der Freihafen 3 ( 1 840), H. 3, S. I 57- I 77, hier: S. I 24, I 54, I 57-1 63 und I 66 f.; Dies.: Unvergessenes. Denkwürdigkeiten aus dem Leben von Helmina von Chézy. Von ihr selbst erzählt. Erster Teil. Leipzig I 858 , S. $219,225-230,232$ f. und 24I-244. 
ser hatte sie wohl schon Ende Juni I 802 mit Friedrich Schlegel und 139 Dorothea Veit bekannt gemacht, die etwa Mitte Juni i 802 in Paris eingetroffen und zunächst bei der Kaufmannsfamilie Pobechaim, weitläufiger Verwandtschaft von Dorothea, untergekommen waren.

Der folgende Brief ist der erste bislang bekannte Brief Dorothea Veits an Helmina von Hastfer (die in Z. 5 direkt angesprochen wird). Seine zeitliche Einordnung ist nur auf den ersten Blick klar und macht exemplarisch die Schwierigkeiten bei einer nachträglichen Bestimmung von Schreibdaten deutlich. Der Hinweis auf parallele und dazu >illuminierte Feten < (Z. 9-г I) in Paris und Versailles im Sommer lässt nämlich sofort an den Nationalfeiertag am I 4. Juli I 802 denken; der aber fiel auf einem Mittwoch, so dass der Brief, der von einem » gestern « (Z. 9, I o) spricht, auf den zweiten Blick an einem Donnerstag und nicht »Montag früh «(Z. I ) geschrieben sein müsste. Allerdings dauerten die Revolutionsfeierlichkeiten im Jahre I 802 länger als sonst. Das geht aus einem Bericht Johann Gottfried Seumes hervor, der im Sommer I 802 auf dem Rückweg von Syrakus auch in Paris Station machte und eben jenen Feierlichkeiten mit »Illumination « zum Revolutionstag beiwohnte. Er hält dazu fest: »Die Feier des Tages des Bastillesturms beschloß ein Konzert in den Tuilerien, wo in dem Gartenplatze vor dem Orchester am Schlosse eine unzählige Menge Menschen zusammengedrängt stand. $\ll{ }^{4}$ Das Konzert, auf das sich Seume hier bezieht, war das Wiedereröffnungskonzert der »Chapelle des Tuileries « am Dienstag, dem » 20 . Juli I $802 \ll$, unter »Giovanni Paisiello « ( I 740-I 8 I 6), den Napoleon eigens zur Leitung seines neuen Hoforchesters aus Italien engagiert hatte. ${ }^{5}$ Erst der dritte Blick bringt also an den Tag, dass die anfängliche Vermutung im Kern richtig war, nur dauerte im Juli I 802 die Feier des Revolutionstages mit den obligatorischen Beleuchtungen vom Mittwoch, den I 4. Juli, bis zum Dienstag, den 20. Juli; und der Brief Dorothea Veits an Helmina von Hastfer ist daher mit an Sicherheit grenzender Wahrscheinlichkeit am Montag, dem i 9. Juli i 802, geschrieben worden.

4 Johann Gottfried Seume: Prosaschriften. Darmstadt i 974, S. 573.

5 Annette Monheim: Ein Westfale in Paris: Die Tagebücher des Ludwig Grafen von Bentheim-Steinfurt aus den Jabren I 806/07. Münster 1 997, S. 36. 
140 Dieser Brief präludiert nicht nur wichtige Themen des zeitlich folgenden - bereits gedruckten - Briefes von Dorothea Veit an Helmina von Hastfer vom 25. August i 802 (Wohnungswechsel, Zusammenziehen $^{6}$ ), sondern gibt auch einen einleitenden Hinweis (Z. 4 ff.) auf den schwedischen Gesandtschaftspfarrer Christian Carl Gambs (1 759-1 822), den Helmina von Hastfer früh kennen- und schätzen gelernt hatte und der im April I 804 Dorothea Veit protestantisch taufen und mit Friedrich Schlegel trauen wird.

\section{I.2 Text}

Dorothea Veit an [Helmina von Hastfer]: Paris [vermutlich Montag, Ig. Juli I802]

PARIS Montag früh

Wir haben Sie gestern mit so vieler Gewißheit und dennoch umsonst erwartet, liebe Freundin! wie war's denn, versprachen Sie nicht am Sonntag in Paris zu seyn? ich will nicht hofen daß Sie nur die Kirche und nicht

5 auch die Freunde besuchten; unmöglich kann Helmina Liebe und Freundschaft von ihrem Gottes Dienst trennen! (Nebenher will ich es Ihnen doch nicht verhehlen welch eine Freude ich und Friedrich damit haben, daß Sie so gar nicht von der einseitigen Mode der Aufklärung angesteckt sind.!) Waren Sie wirklich nicht in Paris gestern? haben Sie die Feten nicht Io gesehen, oder hatten Sie eigne in Versailles? Wir waren gestern mehr als je zu hause und einsam. die Ilumination haben wir bloß an den Lampen am gegenüberstehenden Hause bewundert. Friedrich war nicht wohl, wollte nicht ausgehen, und so blieb auch ich in mein Kämmerlein. Aber wissen Sie wohl daß es von Ihnen Pflicht war die Feten zu sehen? wer so gut zu sehen I 5 und so gut zu erzählen weiß als Sie, darf nichts versäumen.

Haben Sie noch nicht wieder auf Mittel gedacht wie wir uns zusammen häußlich einrichten könnten? Unser ganzer Sinn steht jetzt darnach recht viel mit Ihnen | zusammen zu seyn. Diesen und den andern Monat werden wir wohl noch unsre Pension behalten müßen, denn man muß immer einen

6 Vgl. Ernst Wienecke (Hg.): Caroline und Dorothea Schlegel in Briefen. Weimar I 9 I 4 , S. $353-355$. 
20 Monat vorher bezahlen und aufsagen. Aber alsdenn werden wir auf keinen 141 Fall länger bleiben. Denken Sie sich ja etwas hübsches für unser Zusammenseyn aus! Liebe Helmina, es ist vielleicht Ihnen nicht eben solch Bedürfniß mit mir zu seyn, als es mir ist mit Ihnen zu seyn - Ich bin Ihnen recht herzlich gut - und habe mir schon in Gedanken allerley reizende

25 Abende ausgedacht die wir zusammen im winter mit vaterländischer Poesie zu bringen wollen; und im Herbst mit kleinen Fußreisen und Spaziergängen, und wie wir alles gemeinschaftlich treiben, und uns alles mittheilen wollen - wenn es nur wahr würde! aber leider habe ich in meinem Schicksale etwas ähnliches vom MALENCONTREUX der MADAME

30 GENLIS - meine schönen Bilder der Zukunft wollen sich selten zur Wirklichkeit bringen lassen - ich schließe mich aber immer nur um so inniger an die Fantasie, und lasse diese meine Wirklichkeit seyn, anstatt mich einer wirklichen Wirklichkeit sklavisch zu verkaufen, die mir nun einmal nicht ansteht - und darum nennen meine Freunde mich eine

35 Phantastin - könnten Sie liebe Helmina mit dieser gutmüthigen MALENCONTREUSE | gern leben? -

Wir wissen nicht ein Wort von Schweighäuser, war er in VERSAILLES? ist er seit den Donnerstag da geblieben? wir hoften ${ }^{\sim}$ sehr ihn zu sehen - ich möchte die Leute denen ich gut bin immer in meiner Nähe wissen, oder 40 doch wenigstens etwas von ihnen hören; darum liebe ich die kleinen Städte so; ich wollte wohl, wir könnten uns in Paris eine Art von kleiner Stadt bilden von lauter Freunden, wo wir denn die Neuigkeiten der großen Stadt um uns bloß wie durch Zeitungen erführen - Sehen Sie wieder die Phantastin!

45 Lassen Sie uns recht bald von Ihnen hören theure Helmina! oder besser noch lassen Sie uns bald Sie sehen. Schreiben Sie uns ein freundliches wort, wie es Ihnen ergangen ist, und was Sie vorhaben.

Ihre Dorothea Schlegel.

Friedrich legt sich Ihnen zu Füßen, und grüßt Sie herzlich und freundlich. so Wollen Sie uns dem Grafen d' Escherny empfehlen? 
6 Nebenher $x x x\lceil$ will $\rceil$ ro oder ha $\langle\mathrm{d}\rangle\langle$ tten; 23 Bedürfniß $\lceil$ mit mir zu seyn $\rceil 33$ mich 〈in〉 33 Schweighäuser] Schweigh (Suspension als äuser aufgelöst) $33 \mathrm{er}$ 〈scho 〉 49-50 Von Friedrich bis empfehlen?] eigenhändige Nachschrift D. Veits.

\section{ÜBERLIEFERUNG}

H Biblioteka Jagiellońska, Kraków, Slg. Varnhagen (o. Sign.). - I Doppelbl., 3 S., Tinte, o. Adr., o. Dat.

2. Friedrich Schlegel an Helmina von Hastfer; vermutlich zwischen dem I. und I o. November I 802

\section{I Erläuterung}

Dass Helmina von Hastfer auch die Adressatin des zweiten bisher unbekannten Briefes ist, erkennt man vor allem daran, dass Friedrich Schlegel sie an ihr »Versprechen « erinnert, einen Beitrag für sein $\gg$ JOURNAL « (Z.9), die Europa, zu liefern. Sie hatte allerdings ihre Beiträge für den ersten Band unter der Rubrik »VII. Ansichten und Miscellen « schon vor dem 22. Oktober I 802 bei ihm abgegeben, ${ }^{7}$ denn an diesem Tag schickte Friedrich Schlegel sein erstes Manuskriptpaket an seinen Verleger Friedrich Wilmans, das auch diesen Beitrag enthalten haben muss (s. 3); der Wunsch wird sich also auf weitere Beiträge beziehen. ${ }^{8}$

Ferner setzt das Verständnis dieses Briefes die Kenntnis eines Bedenkens voraus, das Friedrich Schlegel in einem Schreiben vom 20. September 1802 an Johann Gottlieb Schweighäuser, das an die Anschrift von dessen Hausmeisterstelle beim Grafen Voyer d'Argenson in Ormes (Poitou) adressiert ist, äußert. Schlegel schreibt dort, dass er und Dorothea sich Sorgen wegen des »Alleinwohnens « Helmina von Hastfers machten, da sie dadurch aufgrund der »Pedanterie der Pariser « in einen schlechten Ruf geraten

7 Vgl. dazu: Europa. Eine Zeitschrift. Herausgegeben von Friedrich Schlegel bei Friedrich Wilmans [Nachdruck der Originalausgabe. Darmstadt I963]. Bd. I/ I, S. I 59- 170.

8 Nach $\mathrm{KFSA}_{3}$, S. XXIII stammt wohl der Beitrag > Guther Rat für Reisende < in der Europa, Bd. I/2, S. I 45 f., von Helmina von Hastfer. 
könnte. ${ }^{9}$ Daran schließt der zentrale Satz des vorliegenden Briefes 143 an: »Hier bietet man Ihnen jezt ein sehr hübsches Apartement mit der Pension für 7 LOUISD. monatlich an. «(Z. I 2$)$ Friedrich Schlegel und Dorothea Veit hatten sich also zwischenzeitlich auch selbst um eine Unterkunft für Helmina gekümmert.

Helmina von Hastfer, die aufgrund der Herausgabe der Französischen Miscellen im Oktober 1802 wieder in Paris sein musste, suchte spätestens ab Ende September eine neue Wohnung und zog dann möglicherweise zunächst in die alte Wohnung Johann Gottlieb Schweighäusers ein. Bald schon folgte sie aber dem Rat des Pariser Publizisten Charles Vanderbourg (1765-1 827), einem Freund Schweighäusers, und begab sich wohl spätestens ab Mitte November I 802 in »Pension « (Z. I 2) bei dem deutschen Buchhändler Henrichs, wo Johann Friedrich Reichhardt sie im Dezember I 802 besuchte. ${ }^{10}$

Für die Bestimmung des Schreibdatums kommt aber als entscheidende Determinante hinzu, dass in dem Brief der »Bürger Schweighäuser « als schon wieder in Paris anwesend genannt wird (Z. 2 I ), was erst im Winter, d. h. wohl ab November i 802, der Fall war. Da Helmina von Hastfer für einen »Sonntag « (Z. . $)$ eingeladen wurde, können Friedrich Schlegels öffentliche > Sonntagsvorlesungen < über Deutsche Literatur im WS I 802/ I 803, die nicht vor Mitte November begonnen haben dürften, noch nicht aufgenommen worden sein; ebenso wie Helmina von Hastfers Umzug in die Familie Henrichs bis Mitte November wohl noch nicht vollzogen war. Daraus lässt sich zusammenfassend schließen, dass der Brief zwischen dem I. und dem Io. November I 802 entstanden sein wird.

Was im übrigen Schweighäusers »sehr nomadische Lebensart « (Z. 2 I) betrifft, so mag sich diese auf seine wiederholten Wohnungswechsel in Paris beziehen. Helmina von Hastfer könnte zunächst in seiner Wohnung gewohnt haben - das Verlobungsverhältnis bestand wohl nach der Rückkehr von Ormes nicht mehr. Angesichts der dürf-

9 Vgl. Robert L. Kahn: »Fifteen Letters from Friedrich and Dorothea Schlegel to J. G. Schweighäuser, Paris, I $802-1804 \ll$. In: PMLA 75 (1960), Nr. 3, S. 197-2 I 5 , hier: S. 208.

10 Vgl. Johann Friedrich Reichardt: Vertraute Briefe aus Paris I 802/I803. Berlin I 98 I, S. 92 . 
144 tigen Quellenlage ist es jedoch auch nicht ausgeschlossen, dass Johann Gottlieb Schweighäuser in der ersten Novemberhälfte wegen einer pflegebedürftigen Erkrankung zwischen Paris und Straßburg pendelte. ${ }^{11}$

\subsection{Text}

Friedrich Schlegel an [Helmina von Hastfer]: [Paris, vermutl. zwischen dem I. und Io. November I 802]

\section{Freitag Vormittag}

Ich war neulich dadurch daß Sie uns wieder mein Vermuthen so schnell von den Augen verschwanden, so überrascht, daß ich vergaß Sie zu bitten, ob Sie nicht, wenn Sie auf einen Tag hereinkommen, uns diesen ganz

5 schenken und bei uns essen wollten: Da Sie den Sonntag nannten so werden wir Sie diesen Tag erwarten wenn Sie es nicht noch anders bestimmen.

Haben Sie Musse und ist ihre Stimmung günstig ${ }^{\sim}$, so denken Sie an mich, ich meine an Ihr Versprechen und mein JourNal. Haben Sie hier etwas zu Io bestellen oder einzurichten, so werden wir gern alles thun, besonders um Sie in unsre Nähe zu bringen. | Hier bietet man Ihnen jezt ein sehr hübsches Apartement mit der Pension für 7 LOUISD. monatlich an. Es wäre ein Gewinn für uns, wenn Ihre Verhältnisse Ihnen erlaubten, dieses Anerbieten anzunehmen.

I 5 Vor allen Dingen erhalten Sie uns Ihre Freundschaft u Andenken und kommen Sie den Sonntag nicht zu spät

Ihr ergebenster

Friedrich Schlegel.

11 Vgl. dazu den wahrscheinlich ebenfalls frühestens Anfang November r 802 entstandenen Brief Dorothea Veits an Helmina von Hastfer: »Il Povero Schweighäuser ist krank, wir haben diesen Morgen Nachricht von ihm gehabt. wie kann er krank seyn, da Mutter und Tochter ihn so lieben? Aber im Ernst, er muß doch recht krank seyn, da er sich beklagt, und den Arzt verlangt.«Wienecke (Hg.): Caroline und Dorothea Schlegel in Briefen (s. Anm. 6), S. 361 f. »Tochter « bedeutet hier Schwester; Mutter und Schwester lebten in Straßburg, wo Johann Gottlieb Schweighäusers Vater an der Universität als Altphilologe tätig war. 
Daß meine Frau sich Ihnen aufs beste empfiehlt, versteht sich ohnehin, da 145 20 ich in unserm gemeinschaftlichen Namen gesprochen habe.

Der Bürger Schweighäuser führt jetzt eine sehr nomadische Lebensart; er ist etwas REPANDIRT, doch sahen wir ihn gestern einen Augenblick

\section{APPARAT}

Is freundschaftliches] freundschaftl (Suspension als iches aufgelöst) 17-20 Von Daß bis Augenblick eigenhändige Nachschrift F. Schlegels 20 gemeinschaftlichen] gemeinschaftl (Suspension als ichen aufgelöst).

\section{ÜBERLIEFERUNG}

H Biblioteka Jagiellońska, Kraków, Slg. Varnhagen (o. Sign.). - I Doppelbl., 3 S., Tinte, o. Adr., o. Dat.

3. Friedrich Schlegel an Friedrich Wilmans, 22. Oktober I 802

\section{I Erläuterung}

Als Friedrich Schlegel am 22. Mai I 802 an Ludwig Tieck schreibt: »Ich habe mit Wilm[ans] etwas ganz leidlich gemacht, nämlich die Europa bei ihm angebracht $\ll,{ }^{12}$ ahnt er wohl noch nicht, dass damit bis Oktober I 805 ein durchaus kontrastreicher und von Höhen und Tiefen geprägter Briefwechsel dieses »JOURNAL « (Z. I 2) begleiten würde, dessen erster (negativer) Höhepunkt schon am 3 I.I 2.I 802 (s. 4) erreicht ist.

Nach einem ersten Schreiben vom I 3. August I 802, welches das Thema Europa nur streift und dabei die rechtzeitige Übersendung der Textmanuskripte in Aussicht stellt, ${ }^{13}$ liegt dem bislang unveröffentlichten Brief vom 22. Oktober I 802 endlich ein $\gg$ Packet «, wie es später heißt, ${ }^{14}$ mit Drucktexten für das $\gg$ I $\underline{\text { te }}$ Stück « (Z. 72) des Ersten Bandes der Europa bei. Immerhin fehlen zu diesem Zeitpunkt,

12 KFSA 25, S. 362.

13 Briefe von und an Friedrich und Dorothea Schlegel. Gesammelt und erläutert durch Josef Körner. Berlin 1926, S. 42-44, hier: S. 42: »Das Mscrpt erhalten Sie gewiß vollständig zur bestimmten Zeit [...].«

14 Friedrich Schlegel an Friedrich Wilmans, 26. November I 802. In: Briefe von und an Friedrich und Dorothea Schlegel (s. Anm. I3), S. 45. 
146 wie aus dem folgenden Briefwechsel hervorgeht, noch sich anschlieBende »Restanten « (Z. 74): Friedrich Schlegels Aufsatz Litteratur sowie sein Essay Nachricht von den Gemählden in Paris. Der Beitrag Litteratur erreicht Wilmans mit Brief vom 26.I I.I 802; der erste Teil des Gemählde-Essays wird zusammen mit dem Brief vom 20.I 2.I 802 übermittelt; ${ }^{15}$ der zweite Teil folgt erst mit dem hier als vierter Brief abgedruckten Schreiben vom Jahresende (4.2, Z. I) - obwohl bis Ende des Jahres das » I $\underline{\text { te }}$ Stück « doch bereits gedruckt sein sollte, damit im Januar I 803 die Auslieferung beginnen konnte. ${ }^{16}$

Neben den Routinethemen des Briefes, die drucktechnische und graphische Fragen behandeln und zu denen bei Friedrich Schlegel immer wieder auch Geldfragen, insonderheit die Begleichung seiner $\gg$ Auslagen « (Z. 26) und Schulden (Z. 29) durch Dritte (Z.30) gehören, ${ }^{17}$ fallen zwei Besonderheiten auf. Dazu gehört erstens der Hinweis auf einen angeforderten, aber nicht erhaltenen »physi-

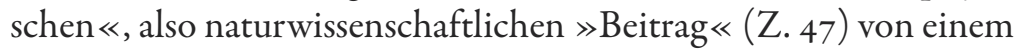
Dr. Johann Jakob Casimir Buch (1778-185 I), einem Frankfurter Apotheker und Arzt aus dem Bekanntenkreis Clemens Brentanos. ${ }^{18}$ Friedrich Schlegel hatte Buch vermutlich erst während seines kurzen Aufenthaltes in Frankfurt Anfang Juni I 802 kennengelernt. ${ }^{19}$ Buchs Beitrag sollte einen offenbar einschlägigen des berühmten Jenenser Physikers Johann Wilhelm Ritter (I 776- I 8 Io) ersetzen, zu dem Friedrich Schlegel ab Mitte I 802 anscheinend keinen Kontakt mehr suchte, obwohl er in Jena eng mit diesem befreundet gewesen war. ${ }^{20}$ Die zweite Besonderheit ist ein Hinweis auf die erste Vertonung eines

15 Vgl. Friedrich Schlegel an Friedrich Wilmans, 26. November i 802. In: Briefe von und an Friedrich und Dorothea Schlegel (s. Anm. I 3), S. 45 und 47.

16 Friedrich Schlegel an Friedrich Wilmans, 20. Dezember I 802: »Sehr muß ich mich wundern, daß Sie das I te Heft nun nicht im Januar versenden zu können glauben.« Briefe von und an Friedrich und Dorothea Schlegel (s. Anm. I 3), S. 47.

$17 \mathrm{Zu}$ den Schulden bei Dr. Buch vgl. schon den Brief an Wilmans vom I 3. August I 802; Briefe von und an Friedrich und Dorothea Schlegel, (s. Anm. I 3), S. 43.

$18 \mathrm{Zu}$ Johann J. C. Buch vgl. Clemens Brentano: Werke und Briefe. Frankfurter BrentanoAusgabe. Hg. von Anne Bohnenkamp-Renken u. a. Bd. 38, r. Stuttgart 2003, S. 436.

19 Vgl. KFSA 25, S. 673.

20 Vgl. zum Verhältnis Friedrich Schlegels zu Johann Wilhelm Ritter KFSA 25, S. 172 und I 97. - Zu seinem späteren, nicht erhaltenen und nie beantworteten Briefwechsel mit den Schlegels in Paris vgl. M. C. Harding (Hg.): H. C. Örsted avec divers savants. Bd. 2. Kopenhagen I 920, S. 27, 40 f., 43, 5 I, 57 und 207. 
Schlegelgedichtes durch Wilhelm Schneider (I 78 I-I 8 I I). Es han- 147 delt sich dabei um Schlegels $\gg$ Lied « (Z. 59) aus dem Musenalmanach für das Jahr $1802 .{ }^{21}$ Die Vertonung ist dann aber nicht in der Europa erschienen, wie Schlegel es wünschte (Z. 60), sondern im Musikalischen Taschenbuch der Gebrüder Werden für das Jahr I 803 , für das sie am I 5 . September I 802 in der Allgemeinen Literaturzeitung bereits angekündigt worden war. ${ }^{22}$ Rätselhaft bleibt indes, wie Friedrich Schlegel zwischenzeitlich in den Besitz dieser Vertonung eines eigenen Gedichts gelangen konnte - es sei denn, es hätte sich gar nicht um jenes $\gg$ Lied $\ll$ gehandelt.

\subsection{Text}

Friedrich Schlegel an [Friedrich Wilmans]: Paris, Freitag, 22. Oktober 1802

Paris. den $22 \stackrel{\text { ten }}{=}$ October 1802 .

Hier übersende ich Ihnen, werthester Freund, das Mscrpt zum ersten Stück der Europa, bis auf einige Restanten, von denen nachher ein mehres.

Nun hab' ich zwei große Bitten an Sie. Fürs erste, die sorgfältigste genauste Correctur. Zweitens, wählen Sie das format ja nicht zu groß. Ich 5 sehe wohl, daß die Hälfte zum wenigsten, oft auch wohl Drei Viertheil der Arbeit auf mich fallen wird; so leicht es mir aber auch wird, bei den vielen neuen und intressanten Gegenständen die ich hier vor Augen habe, fleißig zu sein, so ist es mir doch nicht recht möglich, meine Schreibart zu ändern,

Io [die] nun einmal sehr CONÇIS und kurz [xxx]; ich kann eher alles andre sein, nur nicht weitläuftig. Uebrigens können Sie meines Eifers und meiner Sorgfalt für das Fortkommen dieses JourNaLS gewiß sein; versprechen | Sie nur ja aufs ausdrücklichste, daß die 4 Stücke jährlich ganz pünktlich erscheinen sollen; ich halte gewiß Wort, an der Nichterfüllung dieser

I 5 Bedingung sind mehre gute Journale gescheitert. - Für den Fortgang des JournaLs könnt' es vielleicht sehr vortheilhaft sein, wenn Sie Sich entschließen wollten, zu jedem Stük, oder doch zu jedem Bande einen

21 Vgl. Lied (Schaff das Tagwerk meiner Hände). In: KFSA 5, S. I 54.

22 Intelligenzblatt der Allgem. Literatur-Zeitung, Nr. I63, I 5. September I 802, Sp. I 44. 
Kupferstich zu geben; ich wollte schon zweckmäßige Gemählde aus der Gallerie aussuchen, man kann hier eine gute Zeichn[ung] von solchem 20 Umfange für 2 französ ${ }^{\sim} \mathrm{LD}[\mathrm{RS}][\mathrm{h}]$ aben! Wollten Sie zu jedem Stück ein[en] $[\mathrm{K}]$ upfer geben, so könnte man bisweilen auch zur Abwechslung eine von den hier immerfort erscheinenden Carricaturen nehmen; das würde wohlfeiler sein, und ich I wollte sie Ihnen besser aussuchen als sie in dem Journale [in] PARIS und London geliefert werden. Worum ich aber nun

25 recht sehr bitten möchte, das ist, daß Sie mir mit umgehender Post abermals 30 LDRs übersenden möchten, als Auslagen für die Bücher $\mathrm{p}^{\sim}$ aber wenn es sein kann, für das nächstemal zu berechnen aufschöben. Ich bin in der That in Verlegenheit, und werde mit Ungeduld auf die Ankunft Ihrer Assignation warten, da sich grade viel Ausgaben und Verlust 30 gehäuft hat; dagegen aber auch von meiner Seite die pünktlichste Folgeleistung beobachten.

Auch würden Sie mich sehr verbinden, wenn Sie einliegende Briefe

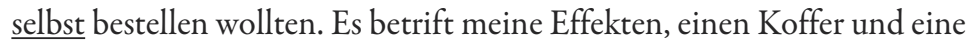
Kiste, die H Dr. J. BuCH mich veranlaßte an die Speditionshandlung des

35 H EMANUel | MülLeR addressiren zu lassen. Nun schreibt man mir von Jena, daß beide Stücke schon am I $2^{\text {ten }}$ Septemb. an benanntes Haus abgegangen. Wie sehr muß ich mich also wundern, daß sie noch nicht hier sind! - Ich bitte Sie recht sehr, Sich dringend danach zu erkundigen; sollte es nöthig sein, diesem Hause die Auslagen gleich zu erstatten, so haben Sie die Güte 40 dieses zu übernehmen, und von der erbetenen Summe abzuziehen. Ich bitte recht sehr Sich dieser Sache anzunehmen. - Dagegen ist es nun nicht mehr nöthig an H DR BUCH die 6 CAROL auszuzahlen, wenn es nicht etwa schon geschehen ist, da ich desfalls eine andre Einrichtung getroffen habe.

Was ein elegantes Aeußre der EUROPA betrift, so verlasse ich $\mid$ mich 45 ganz auf Ihren Geschmack. In Rücksicht des Formats habe ich gerechnet, daß NRO I. etwa 3 Bogen betragen würde.

Von BUCH hatte ich gehoft einen physischen Beitrag noch für das erste Stük zu haben; er ist aber zu sehr mit seinen eignen Angelegenheiten beschäftigt, so daß dieß für jezt nicht zu erwarten ist. Indess. soll die

5o EUROPA darunter nicht leiden; ich werde nicht nur bestimmt für dieses Fach auf eine andre Art sorgen, sondern auch für Beiträge der mannichfaltigsten Art von bedeutenden Schriftstellern in allen Fächern, und sehe überhaupt den glücklichen Erfolg dieser Zeitschrift als ausgemacht an. - 
Beitrag von meinen Freunden aus Deutschland. Einen fast schon fertigen Aufsatz über die Antike habe ich für das 2 뜨 Stück zurückgelegt, weil sonst in dsm I ${ }^{\text {te }}$ Stück fast gar zu viel von bildender Kunst die Rede sein möchte.

Kann die beigelegte Musik für das kleine Lied mit abgedruckt 60 werden, so würde es mir angenehm sein, und vielleicht auch mehren Lesern.

Ich wünsche daß es Ihnen | und den Ihrigen recht wohl gehen mag. Ihr ergebenster Fr. Schlegel.

65 Haben Sie Nachricht - ob meines Bruders Ion gedruckt - oder Fichte's neue Darstellung der Wissenschaftslehre erschienen ist, so lassen Sie es mich ja wissen

P. S. Sie erhalten das Mscrpt. was der Inhalt anzeigt, hier vollständig, ausgenommen NRO II - III und IV. - | NRO III wird kurz sein, NRO IV nur 70 ein paar Seiten, zur Ankündigung, NRO II aber - die Beschreibung der Italiän Gemählde hier - wird ein langer Aufsatz; sollten Sie finden, daß mehr Mscrpt da sei, als zum I $\stackrel{\text { ten }}{ }$ Stück nöthig, so kann dieser Aufsatz abge | brochen werden, und ich werde anzeigen, wo dieß am schicklichsten geschehen kann. - Sie erhalten diese Restanten spätestens in 8-1o Tagen.

$75-$

\section{APPARAT}

10 [die] (Textverderbnis d. Tintenfleck) 10 [xxx] (Textverderbnis d. Tintenfleck: Es können ca. drei Grapheme verlorengegangen sein). 14 mehre $\langle\mathrm{J}\rangle \mathbf{1} 9$ Zeichn[ung] (Textverderbnis d. Tintenfleck) 202 [französ ${ }^{\sim} 20 \mathrm{LD}[\mathrm{RS}]$ (Textverderbnis d. Tintenfleck.) $20[\mathrm{~h}] \mathrm{aben}$ (Textverderbnis d. Tintenfleck) 20 ein[en] (Textverderbnis d. Tintenfleck) $2 \mathbf{2}[\mathrm{K}]$ upfer (Textverderbnis $d$. Tintenfleck). 23 wollte $\langle\mathrm{S}\rangle^{\prime}$ sie $23 \mathrm{f}$. in 「dem JournaLE $\rceil 24$ [in] (Schreibversehen, Flüchtigkeit: in ergänzt) 25 möchte, da $\langle\beta\rangle^{\prime} s 25$ daß $\langle s\rangle^{<}$Sie 30 Seite $\mathrm{d}\left\langle\right.$ e $><$ ie 35 man $\left\langle\right.$ hier $\left.{ }^{\sim}\right\rangle 42$ die $\langle 5\rangle<6426<$ LDRS $>53$ Zeitschrift $\left\langle\right.$ schon $\left.{ }^{\sim}\right\rangle 59$ die $<\mathrm{Mu}>68$ anzeigt, $\lceil$ hier $\rceil 65-74$ Von Haben bis Tagen. - ] eigenbändige Nachschriften F. Schlegels.

\section{ÜBERLIEFERUNG}

H Klassik Stiftung Weimar/Goethe-Schiller-Archiv, Hs. 96/2549. - 2 Doppelbl., 8 S., Tinte, o. Adr. - Bl. I Tintenfraß im Tintenklecks, dadurch Ausbruch einer Stelle im unteren Drittel des Textes mit Textverlust recto und verso. 


\section{I Erläuterungen}

Dem zweiten noch unveröffentlichten Schreiben Friedrich Schlegels an Friedrich Wilmans vom Jahresende i 802 geht nach dem 22. Oktober d. J. ein sich - allerdings einseitig - beschleunigender Briefwechsel voraus. Friedrich Schlegel schreibt zunächst etwa vier Wochen später (am 26. November I 802) unter Beilage seines noch ausstehenden Aufsatzes Litteratur »in der ängstlichsten und peinlichsten Besorgniß«, weil er von Wilmans noch keine Eingangsbestätigung seiner Manuskriptsendung in Händen hält. ${ }^{23}$ Weitere rund vier Wochen später, am 20. Dezember I 802, hat Wilmans zwar zwischenzeitlich geantwortet - der nur zu erschließende Brief datiert vermutlich aus dem Zeitraum zwischen dem 26.I I. und dem I 5. I 2. 1802 -, aber die Antwort kam nach Schlegels Ansicht zu spät, sie war nicht explizit genug - so sei der Eingang des Beitrags Litteratur nicht bestätigt worden - und sie habe das Honorar vermissen lassen. ${ }^{24}$ Zugleich wartet Schlegel mit einem delikaten Argument auf: Hätte Wilmans nur früher geantwortet (d. h. wohl auch: Geld geschickt), so hätte er auch früher den noch ausstehenden GemähldeAufsatz erhalten. Dabei muss er im selben Brief zugeben, dass er auch am 20. Dezember nur erst dessen $\gg$ Anfang « übersenden kann. ${ }^{25}$

Wilmans schweigt zunächst beharrlich weiter, bis er endlich am 27. Dezember antwortet; aber das weiß Schlegel Ende des Jahres I 802 natürlich noch nicht. Er schreibt Wilmans daher am 29. Dezember I 802 (Z. I I) einen nicht erhaltenen Brief, und dann noch einmal zwei Tage später den erhaltenen und hier bekannt gemachten vom 3 I.I 2.I 802. Dieser Brief begleitet nicht nur endlich den Schluss des Gemählde-Aufsatzes, sondern demonstriert auch eine bei Friedrich Schlegel in dieser Form wohl nicht ohne Weiteres erwartete und heute peinlich anmutende Aggressivität - die er selbst kurz darauf zur > ängstlichsten und peinlichsten Besorgniß< herunterspielen wird. ${ }^{26}$ Diese

23 Vgl. Briefe von und an Friedrich und Dorothea Schlegel (s. Anm. I3), S. 45.

24 Vgl. ebd., S. $46 \mathrm{f}$.

25 Vgl. ebd., S. 47.

26 So Friedrich Schlegel an Friedrich Wilmans, 7. Januar I 803. In: Raymond Immerwahr: »Bislang unbekannte Briefe Friedrich Schlegels «. In: Jabrbuch des Freien 
Aggressivität gipfelt nicht nur stilistisch in einer (selbst beim besten 151 Willen kaum emendierbaren) Satzellipse (Z. 52 ff.), sondern auch inhaltlich in der ausnehmend starken Behauptung: »Das Unrecht ist so ganz auf Ihrer Seite « (Z.47), mit der er Wilmans alle »Schuld « (Z. 33 ) aufbürdet, während er von sich selbst behauptet: $\gg$ Ich habe alles auf das pünktlichste gethan, was von mir gefordert werden konnte. (Z. 26 f.) Das muss erstaunen, da bereits der Eingangssatz seines Briefes diese Behauptung dementiert.

Wilmans war indessen nicht untätig gewesen; sein am 27. Dezember 1802 geschriebener Brief erreichte Friedrich Schlegel allerdings erst (kurz) vor dem 7. Januar I 803 und glättete vorerst die Wogen: $\gg$ Ihren Brief vom $27^{\text {ten }}$ Dec. habe ich richtig erhalten und freue mich sehr, daß sie alle meine Nachfragen befriedigend beantwortet haben. $\ll{ }^{27}$

4.2 Text

Friedrich Schlegel an [Friedrich Wilmans]: Paris, Freitag, 31. Dezember I 802

Paris den $3 \mathrm{I}^{\text {ten }}$ December 1802

Hier erhalten Sie den Schluß des Aufsatzes über die Gemählde. Ich bin sehr erstaunt darüber, daß Sie noch den Druck nicht hatten anfangen lassen

5 bei Ihrem lezten Schreiben. Ich hoffe gewiß, es ist unterdessen geschehen, und Sie werden nun das durch Ihre Schuld entstandene Versäumniß so viel als möglich wieder gut machen. Ich rechne darauf, daß das I트 $\mathrm{Heft}$ noch am Schluß des Januars versandt werde, und ich mache dieß zur ausdrücklichen Bedingung, eben so wohl als daß Sie mir mit umgehender

I 0 Post den richtigen Empfang melden. Ueberhaupt müssen Sie pünktlich und ordentlicher schreiben als bisher. In Ihrer lezten ist wieder nicht einmal Nachricht, ob Sie meine Sendung vom $26^{\text {ten }}$ November erhalten haben,

Deutschen Hochstifts ( 1967 ), S. 386-405, hier: S. 393: »Sie werden es nicht übel deuten wollen, daß ich in den lezten [Briefen] noch etwas sehr ängstlich geschrieben habe $\ll$.

27 Friedrich Schlegel an Friedrich Wilmans, 7. Januar I 803. In: Immerwahr: »Bislang unbekannte Briefe Friedrich Schlegels « (s. Anm. 26), S. 393. 
nachher hab' ich noch auf den $20^{\text {ten }}$ und den $29^{\text {ten }}$ December an Sie geschrieben.

Bis den 15 ten Januar längstens erwarte ich befriedigende Antwort.

Zeichnungen von den besten deutschen Künstlern die hier sind, und zwar ohne | daß Sie dafür etwas zu zahlen bräuchten, kann ich hier mit Gewißheit versprechen und biete sie Ihnen hiemit. Es könnte gewiß dem Journal sehr vortheilhaft sein.

Mscrpt zum $2^{\text {ten }}$ Stück ist schon vorräthig, mehre wichtige Beiträge liegen bereit; ich werde sie absenden, sobald Sie Ihre Zusage werden erfüllt haben.

Sie scheinen mir zu drohen, daß Sie Ihr feierlich gegebnes Wort nicht halten wollen. - Wie meinen Sie das eigentlich und was glauben Sie für ein

25 Recht dazu zu haben?

Ich habe alles auf das pünktlichste gethan, was von mir gefodert werden konnte. Früher als im October kann man Mscrpt zu einem Journalheft, das im Januar erscheinen soll, doch nicht abschicken. Nachher war es mir aber nicht anzumuthen, meine Mscpt. an einen Mann zu schicken, von dem ich

30 nach seinem Stillschweigen durchaus nichts andres vermuthen konnte, als daß er ge| storben oder doch durch einen andren großen Unglücksfall zu allen Geschäften unfähig sei; eine solche Nachläßigkeit aber als die deren Sie Sich schuldig gemacht, von einem Kaufmann auch nur für möglich zu halten, das konnte mir, wie sie selbst eingestehen werden, gar nicht 35 einfallen.

Zudem wußte ich ja nicht, welches Format Sie gewählt, wie stark Sie auf I Bog ab oder zu das I ${ }^{\text {te }}$ Heft wünschten wie viel Mscrpt. Sie also noch brauchten.

Ich will jeden Buchhändler den Sie wollen zum Schiedsrichter nehmen, 40 ob es nicht (bei dieser Entfernung zumal, und da es gar nichts unerhörtes ist, daß Packete verlohren gehen oder liegen bleiben), ob es nicht Ihre erste und unerlassliche Pflicht mir mit umgehender Post nach Empfang meines ersten Packets zu schreiben.

- Den und hab' ich es erhalten — beifolgendes format denk' ich

45 zu nehmen - so viel Bogen darin beträgt das übersandte Mskrpt — so viel wird es also noch bedürfen u soll' $\left[s^{\sim}\right]$

Das Unrecht ist so ganz auf Ihrer | Seite, das es überflüssig ist, weiter davon zu reden. Das ist aber wirklich gar zu arg, daß Sie noch von Verdruß sprechen, da doch wohl ich derjenige bin der über den Zeitverlust 
so und Verdruß zu klagen hat, den Ihr Nichtantworten, Ihr Nicht 153 druken lassen, und Ihr Nichterfüllen Ihrer Zusagen mir verursacht haben!

Ich gestehe es Ihnen freimüthig, Ich kann Ihr unbilliges Betragen (über das ich besonders in ihrem lezten Briefe nicht wenig erstaunt bin) gar nicht mit ihrer sonstigen Rechtlichkeit zu vereinen weiß; so daß ich auf die

55 Vermuthung gerathen bin, daß irgend ein Verläumder wer weiß durch welche Unwahrheiten Sie gegen mich und mein Unternehmen einzunehmen gewußt. Sagen Sie es mir offenherzig, Sie können gewiß sein, daß ich Ihr Vertrauen auf keine Weise misbrauchen würde.

Es ist recht unangenehm, daß Sie mir den Anfang eines Unternehmens, 60 für das [ich] mit dem größten Eifer gearbeitet habe, und von dem ich mir grosse Wirkungen versprechen darf; so verleidet haben. Ich hoffe Sie werden von nun an ordentlicher und pünktlicher sein.

Ich möchte gern mit Ihnen in gutem Vernehmen bleiben. Machen Sie es mir möglich. Ihr ergebenster

65

Fr. Schlegel

APPARAT

5 nun $\langle$ doch $\rangle$ 17 ich $\left\lceil\right.$ hier ${ }_{23}$ mir $\langle\mathrm{d}\rangle 34$ selbst $\langle$ ebenfalls $\rangle{ }_{36} \mathrm{f}$. Sie $\lceil$ auf I Bogen ab oder zu 737 wünschten $\langle$ stark $\rangle 37$ also $\langle x\rangle 46\left[s^{\sim}\right]$ Vmutl. Textverlust durch Randabschnitt 47 es $\langle$ recht $\rangle$ so $\mathrm{Ihr}\langle$ Nicht $\rangle$ 53 $\mathrm{f}$. ich $\lceil$ besonders in ihrem lezten Briefe 54 sonstigen $\langle$ Ehr $\rangle$ s6 Unternehmen 〈allgem $>60$ [ich] Wortauslassung: Flüchtigkeit $60 \mathrm{mir}\langle$ so $\rangle 63$ nun $\langle\mathrm{xxxxxxxx}\rangle$.

ÜBERLIEFERUNG

H Nachlass Ernst Behler, Fotokopie der HS. - I Doppelbl., 4 S. Tinte, o. Adr. 